\title{
FTIR spectroscopy for detection and identification of fungal phytopathogenes
}

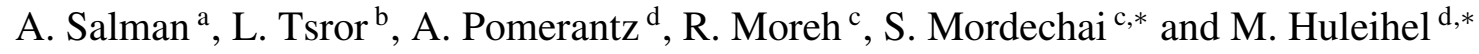 \\ ${ }^{a}$ Department of Physics, SCE-Sami Shamoon College of Engineering, Beer-Sheva, Israel \\ ${ }^{\mathrm{b}}$ Department of Plant Pathology, The Institute of Plant Protection, Agricultural Research \\ Organization, Gilat Experiment Station, Bet Dagan, Israel \\ ${ }^{\mathrm{c}}$ Department of Physics, Ben-Gurion University of the Negev, Beer-Sheva, Israel \\ ${ }^{\mathrm{d}}$ Department of Virology and Developmental Genetics, Faculty of Health Sciences, \\ Ben-Gurion University of the Negev, Beer-Sheva, Israel
}

\begin{abstract}
Soil-borne fungi are considered as major pathogens to many plants and can cause a severe economic damage. Early detection and identification of these pathogens is very important and might be critical for their control. The available methods for identification of fungi like molecular biology, serological tests and PCRs tests (polymerase chain reaction) are time consuming and not always very specific. Fourier-transform infrared (FTIR) attenuated total reflection (ATR) spectroscopy, is considered to be a comprehensive and sensitive method for detection of molecular changes in intact cells.

In the present study we used FTIR-ATR as a sensitive and effective assay for the detection and discrimination between different fungal genera. Our results showed significant spectral differences between the various examined fungi genera. These results proved the possibility of discrimination between these fungi on the genus level.
\end{abstract}

Keywords: FTIR-ATR spectroscopy, fungal detection, fungi, spectral characteristics

\section{Introduction}

Soil fungi are considered as major cause for serious damage to large number of crops resulting significant economical loss $[1,10]$. Early identification enables precise targeting of a pathogen and makes it possible for effective treatment [5]. The differentiation between different fungal species is very difficult by visual or even microscopic inspection. Keys for identifying mycelia are only available for special cases, and frequent identification is practically impossible, especially for closely related species $[11,14]$. Among the techniques offering possibilities for a rapid analysis, molecular biology methods are considered the most rapid and sensitive methods for identification of pathogens, but they are not yet in a large-scale use. Most commercially available identification systems for fungi are based on the physiological characteristics or on serological methods [3,18]. Such identification systems are usually time consuming and not always very specific [25].

Serological tests based on the specificity of the antigen-antibody reaction in the immune systems are not always highly specific. PCRs tests (polymerase chain reaction) are considered the best specific

\footnotetext{
${ }^{*}$ Corresponding authors: M. Huleihel, Department of Virology and Developmental Genetics, Faculty of Health Sciences, Ben-Gurion University of the Negev, Beer-Sheva 84105, Israel. Tel.: +972 8 6479867; Fax: +972 8 6479867; E-mail: mahmoudh@bgu.ac.il. S. Mordechai, Department of Physics, Ben-Gurion University, Beer-Sheva 84105, Israel. Tel.: +972 8 6461749; Fax: 9728 6472903; E-mail: shaulm@bgu.ac.il.
} 
procedures. These two methods are time consuming and expensive. Using these tests it is not possible to diagnose unknown or new pathogens [12], as a result these methods are not suitable for rapidly screening of large numbers of samples.

FTIR spectroscopy is one of the methods that have been successfully used for detecting and identifying microorganisms, especially in food products $[8,12]$. Some of these studies showed that discrimination was possible not only at the genus level, but also at the species [2,13] and strain levels [12,24].

The detection and identification of microorganisms using FTIR spectroscopic techniques is promising as a valuable tool because of its sensitivity, rapidity, low cost and simplicity.

The infrared spectrum of any compound is known to give a unique "finger print" [15]. Together with the large information already known about spectral bands obtained from FTIR spectra of living cells [4], makes FTIR spectroscopy as an attractive technique for detection and identification of pathogens.

\section{Materials and methods}

\subsection{Fungi}

All fungi were supplied by our co-author L. Tsror from the Department of Plant Pathology in Gilat Experiment Station, ARO, Israel. These fungi were originally isolated from diseased plants (stem, root or tuber) of different crops, using classical microbiological techniques [22,23]. The fungi were grown on Potato Dextrose Agar (PDA) (Difco) for several days at $27^{\circ} \mathrm{C}$. Single colony fungal cultures obtained by micromanipulation were maintained in potato dextrose media. Small agar plugs containing the fungus were transferred from a $24 \mathrm{~h}$ fresh Petri dish culture into a liquid medium PDB (potato dextrose broth, Difco). The cultures were grown in continuous shaking for 3-10 days, at $27^{\circ} \mathrm{C}$. Samples were prepared in several batches over a time span of several months. Each pathogen was grown in 4-5 replicates each time.

In this study, we investigated 4 different fungi genera: Rhizoctonia, Colletotrichum, Verticillium and Fusarium oxysporum.

\subsection{Sample preparation}

Samples of these fungi were purified from the media by spinning about $5 \mathrm{ml}$ of medium containing fungi at $2000 \mathrm{rpm}$ for $5 \mathrm{~min}$, washing 4 times with $\mathrm{H}_{2} \mathrm{O}$ and the pellet was suspended in appropriate volume of $\mathrm{H}_{2} \mathrm{O}$ (about $1 \mathrm{ml}$ ).

For ATR examination about $500 \mu \mathrm{l}$ of the above obtained fungal suspension were flattened and spread on the ATR crystal producing a flat layer covering the entire crystal. Then the samples were air dried for about 30 min and examined by ATR spectroscopy.

\subsection{FTIR spectral measurement}

The ATR measurements were performed using FTIR spectrometer (Bruker Tensor 127). Measurements were performed using the FTIR-ATR with a liquid-nitrogen-cooled mercury-cadmium-telluride MCT detector. 128 coadded scans were collected in each measurement in the wavenumbers region 600- 
$4000 \mathrm{~cm}^{-1}$, after the samples were dried. Spectral resolution was set at $4 \mathrm{~cm}^{-1}$. All spectra were baseline corrected and vector normalized using OPUS software.

\subsection{Statistical analysis}

The spectra of various fungi genera were used for subsequent analysis. The Ward [7] minimum variance method was used for the cluster analysis on different regions on the measured spectra. A total of 44 spectra were available for cluster analysis.

\section{Results and discussion}

Figure 1 shows a typical mid infrared absorption spectrum of fungal. The spectrum is dominated by a large absorption band in the lower region at $1050 \mathrm{~cm}^{-1}$. This peak arises mainly from carbohydrate and nucleic acid vibrations [20]. In the higher wavenumbers region, the spectrum is dominated by the water absorption bands at $3350 \mathrm{~cm}^{-1}$. Bands at 2849, 2917 and $3008 \mathrm{~cm}^{-1}$ are mainly due to lipids absorbance $[6,20]$.

All our samples have been dried in a laminar flow chamber using the same procedure. Background spectrum was measured before sample measurement in order to reduce the absorption of water vapor of the atmosphere, and absorption due to other disorders.

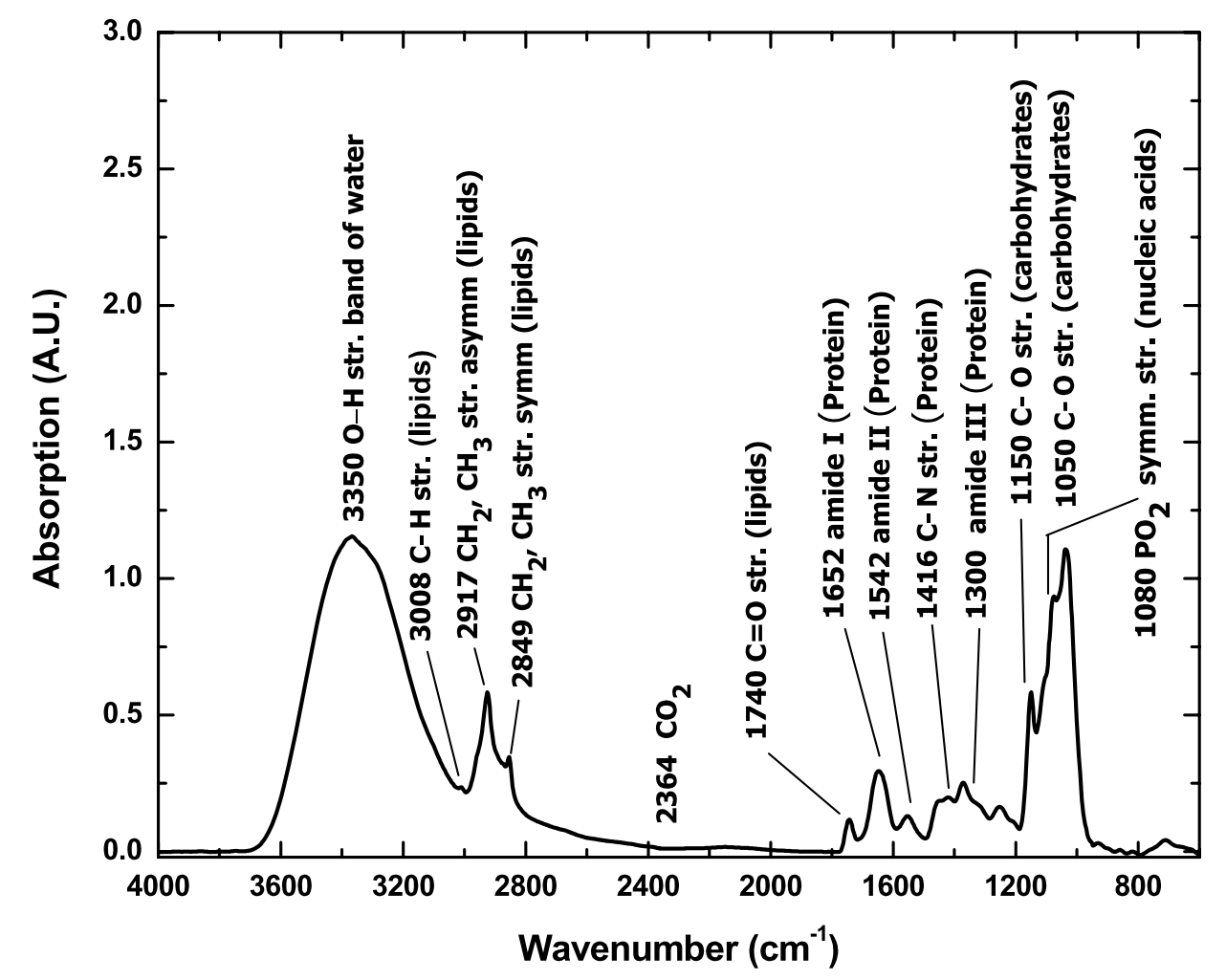

Fig. 1. Typical fungus spectrum in the region $600-4000 \mathrm{~cm}^{-1}$, after baseline correction and vector normalizations. The major absorption bands and their main functional group origin are labelled. 


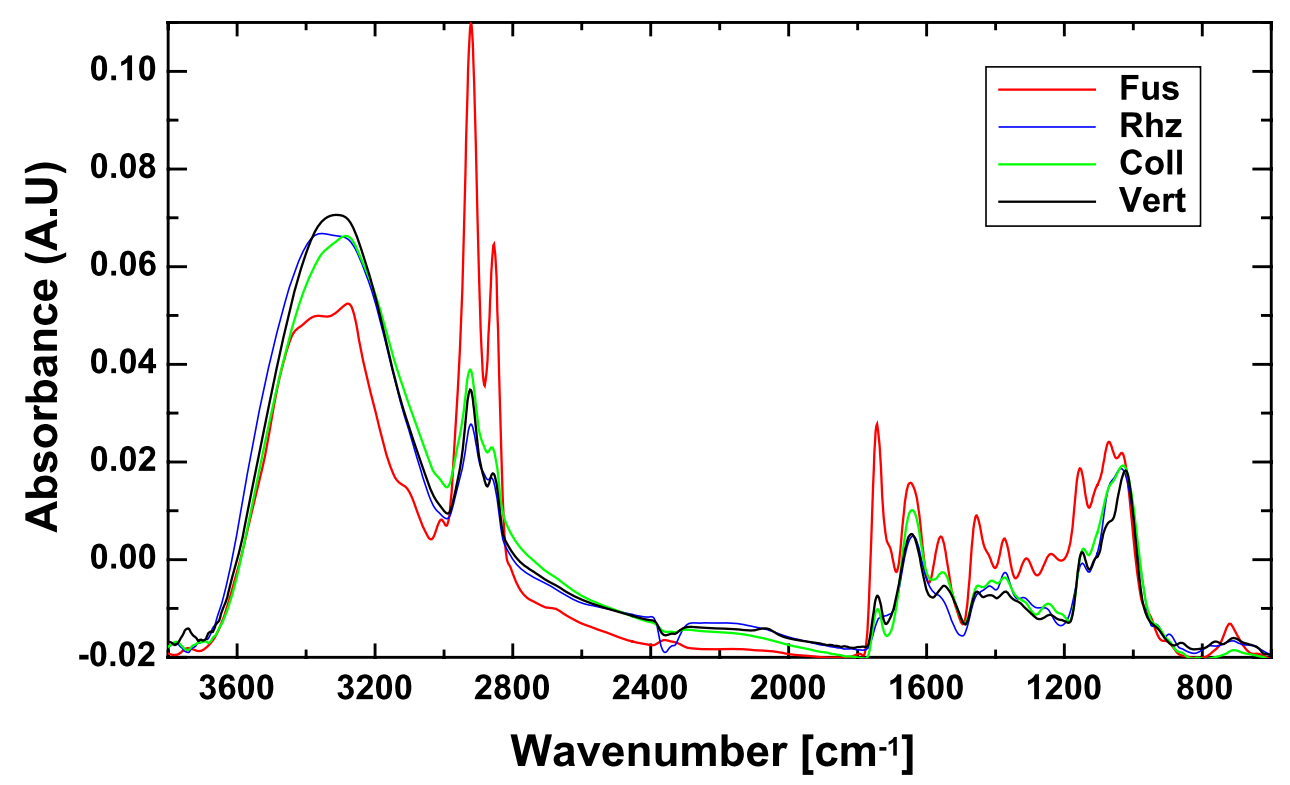

Fig. 2. Mid infrared spectra in the region $600-4000 \mathrm{~cm}^{-1}$ of 4 different genera of fungi: Fusarium, Rhizoctonia, Colletotrichum and Verticillium. (The colors are visible in the online version of the article.)

In addition, each fungus sample examination was repeated at least 10 times at different days and we obtained always consistent spectra, and the same differences among different genera.

After the dehydration process there are still bond water molecules among the samples biomolecules. The contribution of these bonded water molecules are shown in the spectrum in Fig. 1.

Figure 2 presents the spectra of four different fungal genera. There are clear spectral differences between Fusarium genus (red lines) and the others fungi genera. Fusarium genus has intense bands in the lipids region (2800-3020 $\mathrm{cm}^{-1}$ ) and also a large band with centroid at $1740 \mathrm{~cm}^{-1}$ which also arises from lipids absorption [6]. There is a good correlation between these bands in the higher wavenumbers region (2800-3020 $\left.\mathrm{cm}^{-1}\right)$ and the low wavenumber region $\left(1740 \mathrm{~cm}^{-1}\right)$ in the Fusarium genus spectra. The other three genera Rhizoctonia, Colletotrichum and Verticillium have some differences in the lipids, Amide I and Amide II bands.

In order to differentiate among the four fungus genera we used cluster analysis with Ward's algorithm in selected wavenumbers regions. Figure 3(a) shows the clustering using the higher wavenumbers region (2800-3020 $\mathrm{cm}^{-1}$ ), where the lipids are the main contributors for these bands. To make an accurate analysis in this wavenumbers region, all our spectra have been baseline corrected and normalized, then they were bisected in the desired region, background subtracted and the spectra were offset corrected. Thus we insure that all the changes were contributed due to the inherent samples differences.

As can be seen from Fig. 3(a), Fusarium genus can be clearly differentiated from the other genera using cluster analysis in this region. However, it is difficult to differentiate among the other three genera in this region. In the region $1680-1780 \mathrm{~cm}^{-1}$, which is assigned to lipids absorption in the, Rhizoctonia genus can be differentiated from the other two genera Colletotrichum and Verticillium (Fig. 3(b)). Clustering at the carbohydrates region $950-1200 \mathrm{~cm}^{-1}$ was performed on the spectra of Colletotrichum and Verticillium (Fig. 3(c)). This clustering yields a good distinction between these two genera. 


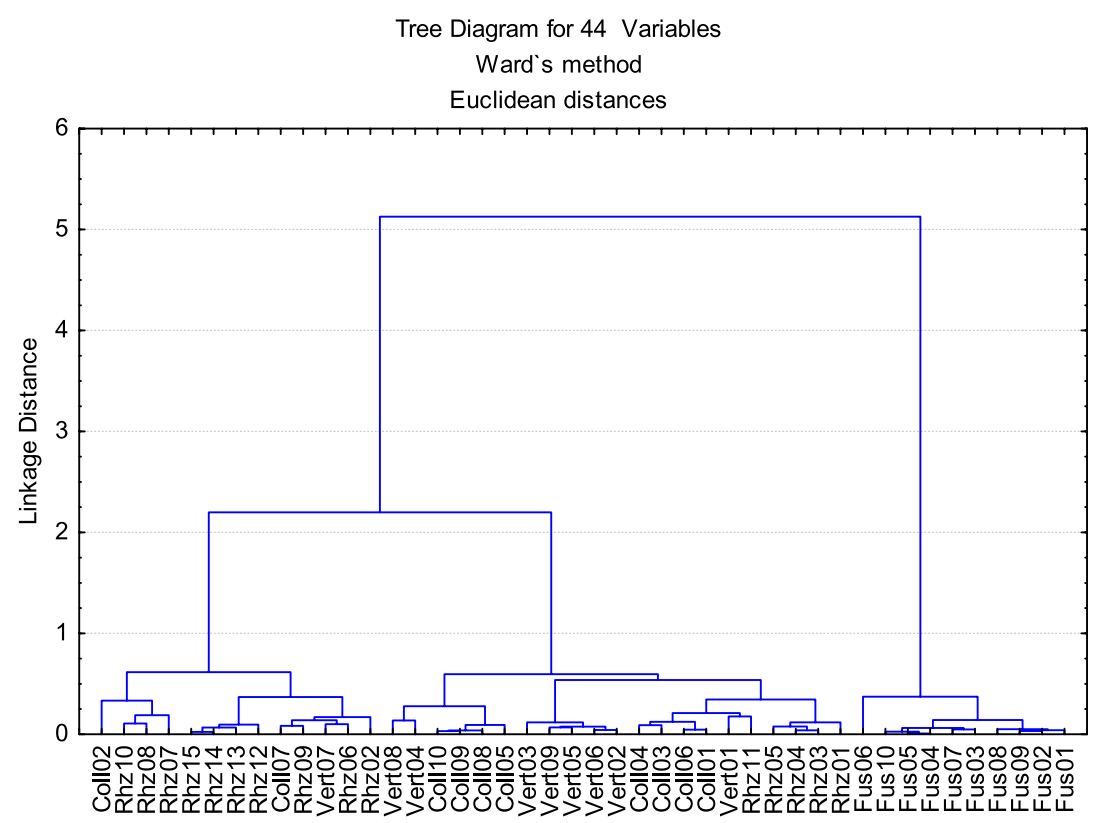

(a)

Tree Diagram for 34 Variables

Ward's method

Euclidean distances

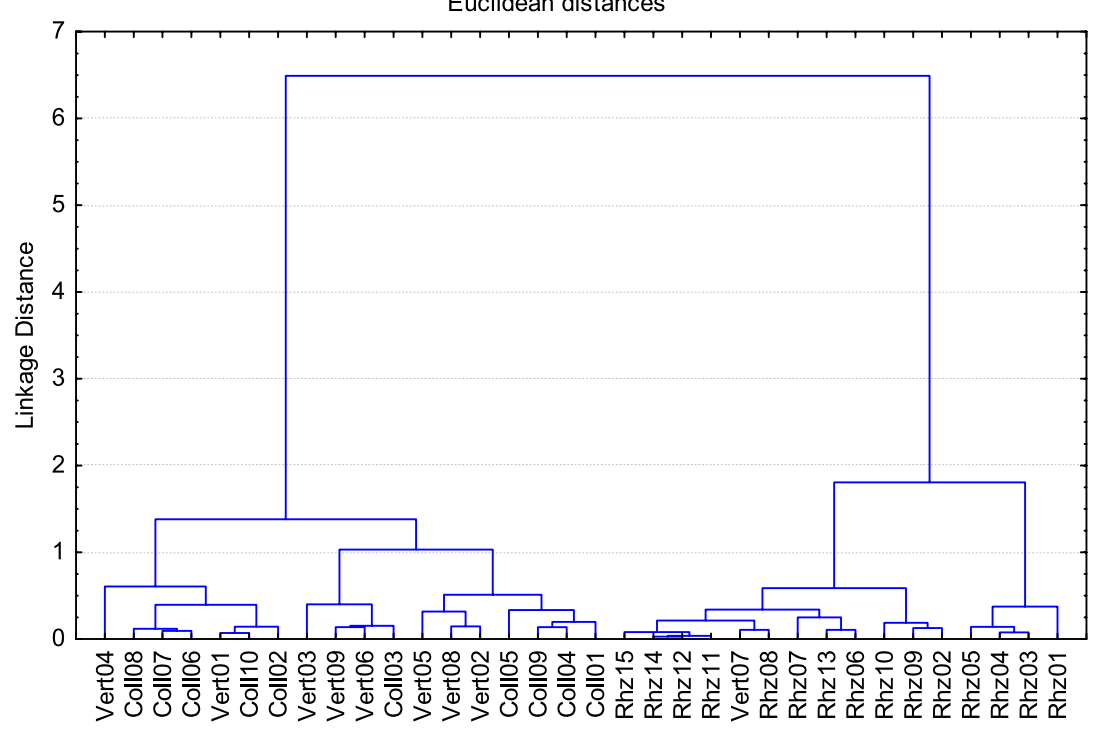

(b)

Fig. 3. Results from cluster analysis on the baseline corrected and vector normalized spectra, in three different spectral regions. (a) Clustering in the region $2800-3020 \mathrm{~cm}^{-1}$ performed on the spectra of the four examined genera. The clustering enables to differentiate between Fusarium and the other phenotypes. (b) Clustering in the range $1680-1780 \mathrm{~cm}^{-1}$ performed on the spectra of Rhizoctonia, Colletotrichum and Verticillium. Rhizoctonia can be differentiated using clustering in this selected region. (c) Clustering in the region $950-1200 \mathrm{~cm}^{-1}$ performed on the spectra of Colletotrichum and Verticillium. The latter shows a good differentiation between the two genera. 


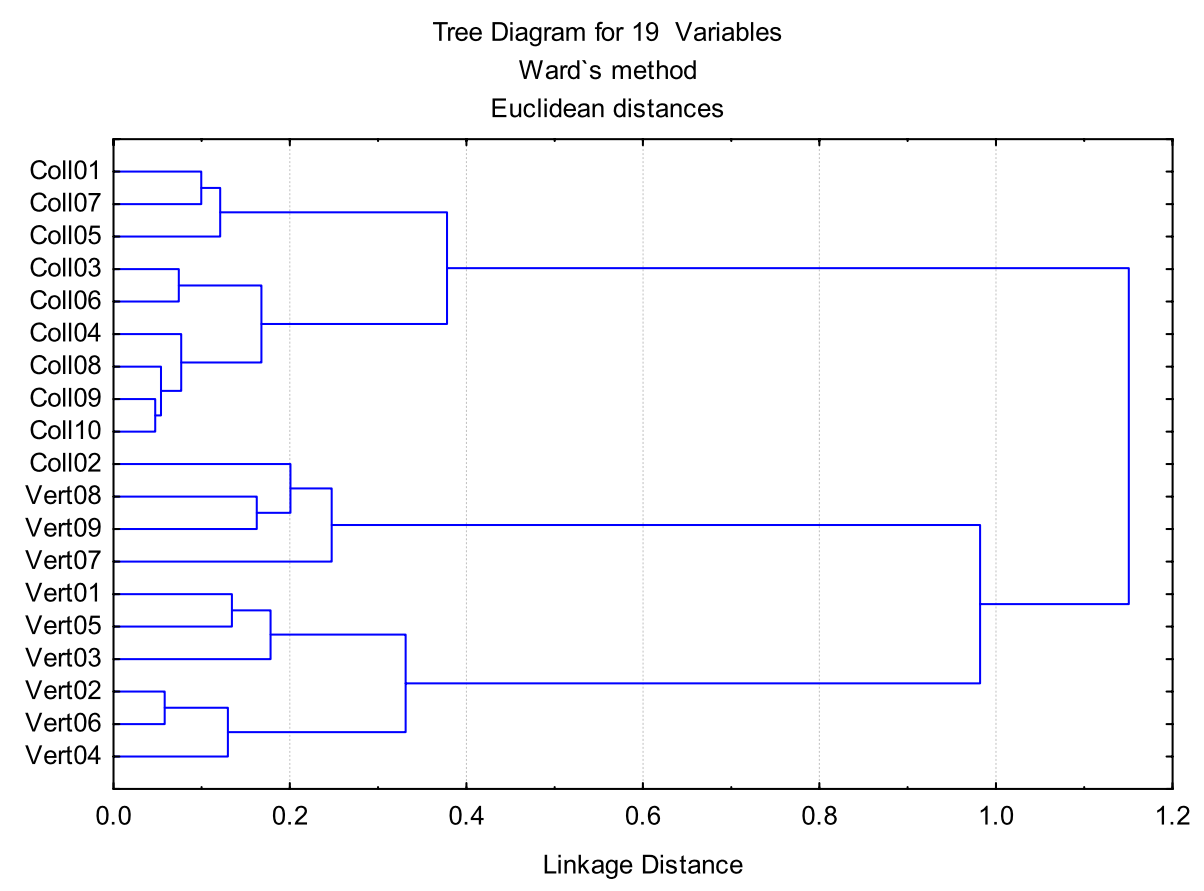

(c)

Fig. 3. (Continued.)

\section{Conclusion}

There is a great potential of FTIR microscopy in tandem with appropriate mathematical tools for an easy and rapid discrimination and identification of various fungi genera, which cause a huge damage in agriculture.

The simplicity of sample preparation, avoidance of chemical (i.e. costs and environmental impact), reliability, short measurement times $(<1 \mathrm{~min})$ compared to other available methods makes FTIR-ATR technique suitable for a large scale screening of fungal samples and for routine analysis.

These facts also encourage the possibility of developing FTIR-ATR spectroscopy as a reliable method for rapid identification of fungal pathogens.

Combining the advantages of FTIR spectroscopy with other used routine techniques, offers the chance to improve the efficiency of fungal classification and identification $[16,17]$. Moreover, the chemical composition of the samples can be simultaneously visualized. Hence, FTIR spectroscopy can help in understanding the complex chemical processes during fungal development and substrate degradation $[9$, $19,21]$.

\section{Acknowledgement}

Financial support by SCE internal research funding is gratefully acknowledged. 


\section{References}

[1] G.N. Agrios, Plant Pathology, Academic Press, New York, 1978.

[2] S.H. Beattie, C. Holt, D. Hirst and A.G. Williams, Discrimination among Bacillus cereus, B. mycoides and B. thuringiensis and some other species of the genus Bacillus by Fourier transform infrared spectroscopy, FEMS Microbiol. Lett. 164 (1998), 201-206.

[3] N.C. Clark, O. Olsvik, J.M. Swenson, C.A. Spiegel and F.C. Tenover, Detection of a streptomycin/spectinomycin adenylyltransferase gene (aadA) in Enterococcus faecalis, Antimicrob. Agents Chemother. 43 (1999), 157-160.

[4] M. Diem, S. Boydston-White and L. Chiriboga, Infrared spectroscopy of cells and tissues: shining light onto a novel subject, Appl. Spectrosc. 53 (1999), 148-161.

[5] G.V. Doern, R. Vautour, M. Gaudet and B. Levy, Clinical impact of rapid in vitro susceptibility testing and bacterial identification, J. Clin. Microbiol. 32 (1994), 1757-1762.

[6] R.K. Dukor, Handbook of Vibrational Spectroscopy, Wiley, Chichester, UK, 2001.

[7] B. Everitt, Cluster Analysis, Wiley, New York, 1980.

[8] M.J. Gupta, J.M. Irudayaraj, C. Debroy, Z. Schmilovitch and A. Mizrach, Differentiation of food pathogens using FTIR and artificial neural networks, Trans. ASAE 48 (2005), 1889-1892.

[9] P. Heraud, S. Caine, G. Sanson, R. Gleadow, B.R. Wood and D. McNaughton, Focal plane array infrared imaging: a new way to analyse leaf tissue, New Phytol. 173 (2007), 216-225.

[10] Y. Katan, Principles in plant pathology, in: Plant Diseases in Israel, J. Rotem, J. Palti and Y. Ben-Yephet, eds, Volcani Center, Bet-Dagan, Israel, 1998, pp. 17-29.

[11] G.-H. Kim, J.J. Kim, Y.W. Lim and C. Breuil, Ophiostomatoid fungi isolated from Pinus radiata logs imported from New Zealand to Korea, Can. J. Bot. 83 (2005), 272-278.

[12] H. Lamprell, G. Mazerolles, A. Kodjo, J.F. Chamba, Y. Noel and E. Beuvier, Discrimination of Staphylococcus aureus strains from different species of Staphylococcus using Fourier transform infrared (FTIR) spectroscopy, Int. J. Food Microbiol. 108 (2006), 125-129.

[13] D. Lefier, D. Hirst, C. Holt and A.G. Williams, Effect of sampling procedure and strain variation in Listeria monocytogenes on the discrimination of species in the genus Listeria by Fourier transform infrared spectroscopy and canonical variates analysis, FEMS Microbiol. Lett. 147 (1997), 45-50.

[14] U. Moreth and O. Schmidt, Investigations on ribosomal DNA of indoor wood decay fungi for their characterization and identification, Holzforschung 59 (2005), 90-93.

[15] D. Naumann, D. Helm and H. Labischinski, Microbiological characterizations by FT-IR spectroscopy, Nature 351 (1991), $81-82$.

[16] A. Naumann, M. Navarro-Gonzalez, S. Peddireddi, U. Kues and A. Polle, Fourier transform infrared microscopy and imaging: detection of fungi in wood, Fung. Genet. Biol. 42 (2005), 829-835.

[17] A. Naumann, S. Peddireddi, U. Kues and A. Polle, Wood production, in: Wood Technology and Biotechnological Impacts, U. Kues, ed., Universitatsverlag Gottingen, Gottingen, 2007, pp. 179-196.

[18] S. Nikkari and D.A. Relman, Molecular approaches for identification of infectious agents in Wegener's granulomatosis and other vasculitides, Curr. Opin. Rheumatol. 11 (1999), 11-16.

[19] K.K. Pandey and A.J. Pitman, FTIR studies of the changes in wood chemistry following decay by brown-rot and white-rot fungi, Int. Biodet. Biodegrad. 52 (2003), 151-160.

[20] S. Sivakesava, J.M. Irudayaraj and C. Debroy, Differentiation of microorganisms by FTIR-ATR and NIR spectroscopy, Trans. ASAE 47 (2004), 951-957.

[21] A. Szeghalmi, S. Kaminskyj and K.M. Gough, A synchrotron FTIR microspectroscopy investigation of fungal hyphae grown under optimal and stressed conditions, Anal. Bioanal. Chem. 387 (2007), 1779-1789.

[22] L. Tsror (Lahkim), O. Erlich, M. Hazanovsky, S. Mordechi-Lebiush and S. Sivan, Aggressiveness of Verticillium dahliae isolates from different vegetative compatibility groups to potato and tomato, Plant Pathol. 50 (2001), 477-482.

[23] L. Tsror (Lahkim) and M. Hazanovsky, Effect of coinoculation by Verticillium dahliae and Colletotrichum coccodes on disease symptoms and fungal colonization in four potato cultivars, Plant Pathol. 50 (2001), 483-488.

[24] T. Udelhoven, D. Naumann and J. Schmitt, Development of a hierarchical classification system with artificial neural networks and FT-IR spectra for the identification of bacteria, Appl. Spectrosc. 54 (2000), 1471-1479.

[25] M. Vaneechoutte and J. Van Eldere, The possibilities and limitations of nucleic acid amplification technology in diagnostic microbiology, J. Med. Microbiol. 46 (1997), 188-194. 


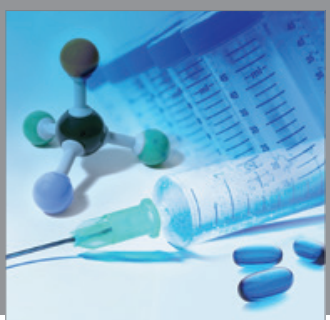

International Journal of

Medicinal Chemistry

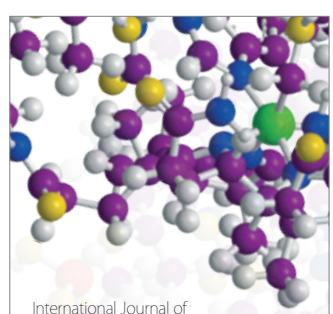

Carbohydrate Chemistry

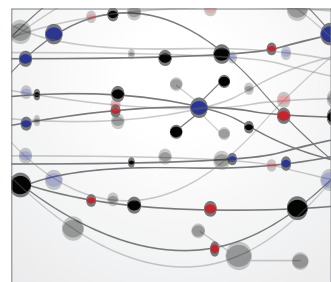

The Scientific World Journal
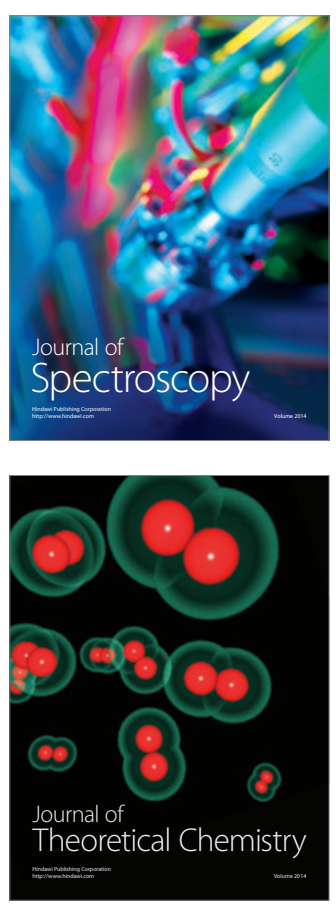
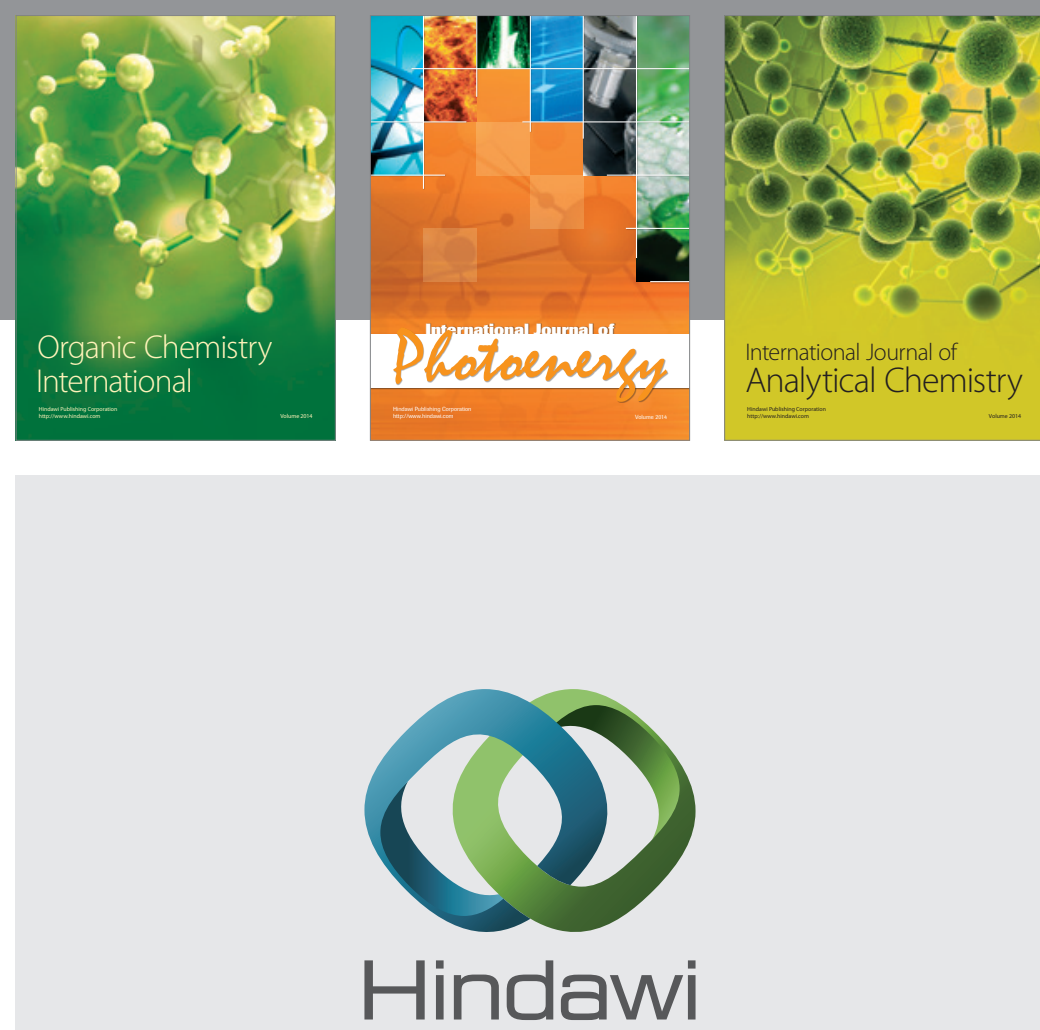

Submit your manuscripts at

http://www.hindawi.com
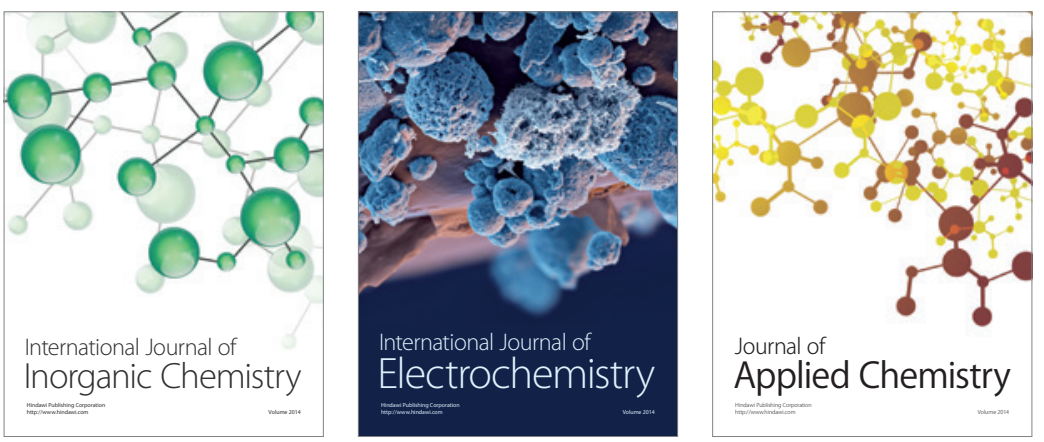

Journal of

Applied Chemistry
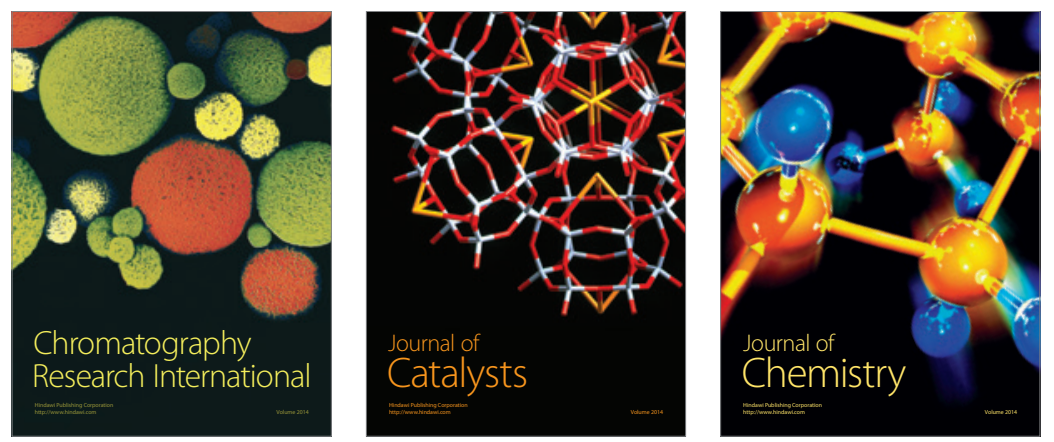
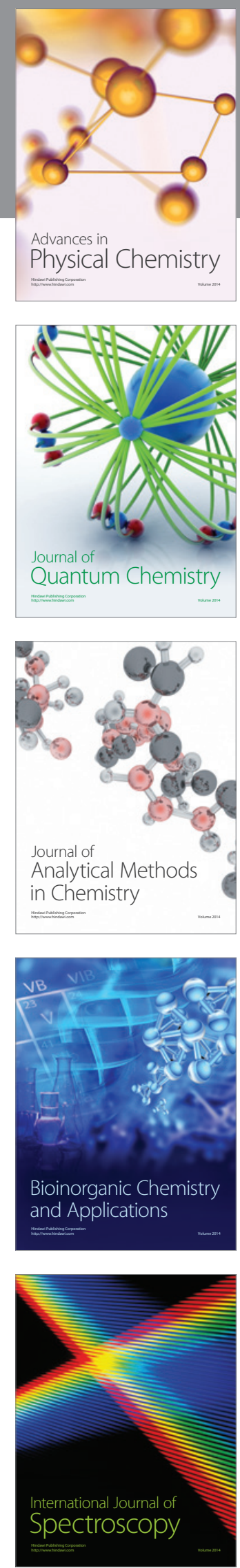